Irish Math. Soc. Bulletin

Number 87, Summer 2021, 45-49

ISSN 0791-5578

\title{
Derangements and Continued Fractions for $e$
}

\author{
PETER LYNCH
}

\begin{abstract}
Several continued fraction expansions for $e$ have been produced by an automated conjecture generator (ACG) called The Ramanujan Machine. Some of these were already known, some have recently been proved and some remain unproven. While an ACG can produce interesting putative results, it gives very limited insight into their significance. In this paper, we derive an elegant continued fraction expansion, equivalent to a result from the Ramanujan Machine, using the sequence of ratios of factorials to subfactorials or derangement numbers.
\end{abstract}

Six students entering an examination hall place their cell-phones in a box. After the exam, they each grab a phone at random as they rush out. What is the likelihood that none of them gets their own phone? The surprising answer - about $37 \%$ whatever the number of students - emerges from the theory of derangements.

We may call any permutation of the elements of a set an arrangement. A derangement is an arrangement for which every element is moved from its original position. Thus, a derangement is a permutation that has no fixed points. The number of derangements of a set of $n$ elements is also called the subfactorial of $n$. Various notations are used for subfactorials: $! n, d_{n}$ and $n$ i are common; we will use ! $n$ (read as 'bang-en').

Dougherty-Bliss and Zeilberger (2020) proved a generalized continued fraction expansion involving Euler's number. They described the occurrence of derangement numbers in the expansion as a "remarkable coincidence", and further commented that "There does not seem to be any immediate combinatorial reason for the derangement numbers to appear." Our derivation in this paper of an expansion for $e-$ equation (7) below - starting from the ratio of factorials to subfactorials, makes the connection clear.

\section{Properties of Derangements}

Derangements were first considered by Pierre Reymond de Montmort. In 1713, with help from Nicholas Bernoulli, he managed to find an expression for the connection between $! n$ and $n$ !. The answer, which he obtained using the inclusion-exclusion principle (Zeilberger, 2008, pg. 560), is

$$
! n=n !\left(1-\frac{1}{1 !}+\frac{1}{2 !}-\frac{1}{3 !}+\frac{1}{4 !}-\cdots \pm \frac{1}{n !}\right)=n ! \sum_{k=0}^{n} \frac{(-1)^{k}}{k !} .
$$

Of course, we see from this that $\lim _{n \rightarrow \infty}(! n)=n ! / e$. In fact, we can write a more precise connection between derangements and arrangements:

$$
! n=\left\lfloor\frac{n !+\frac{1}{2}}{e}\right\rfloor \text {. }
$$

This implies that $! n$ is the nearest whole number to $n ! / e$.

2010 Mathematics Subject Classification. 05A05, 11A55.

Key words and phrases. Continued fractions, Permutations, Derangements.

Received on 22-12-2021; revised 26-5-2021. 
The number $! n$ of derangements of an $n$-element set may be calculated using a secondorder recurrence relation:

$$
! n=(n-1)(!(n-1)+!(n-2))
$$

with $! 0=1$ and $! 1=0$. The subfactorials also satisfy a first-order recurrence relation,

$$
! n=n \times !(n-1)+(-1)^{n}
$$

with initial condition $! 0=1$, which may be compared to $n !=n \times(n-1)$ ! with initial condition $0 !=1$. The first eight values of $! n$ are $1,0,1,2,9,44,265$ and 1854 (for further values, see sequence A000166 in the Online Encyclopedia of Integer Sequences).

The factorial function may be espressed in the familiar integral form:

$$
n !=\int_{0}^{\infty} x^{n} e^{-x} \mathrm{~d} x .
$$

There is a corresponding integral expression for the subfactorial:

$$
! n=\int_{0}^{\infty}(x-1)^{n} e^{-x} \mathrm{~d} x
$$

Expansion of (2) yields de Montmort's result (1). It also allows extension of the subfactorial function to non-integral arguments and analytic continuation to the complex plane.

\section{Continued Fractions and Convergents}

A continued fraction expansion of an irrational number $x$ is written, in expanded form (centre) and concise form (right), as

$$
x=a_{0}+\frac{1}{a_{1}+\frac{1}{a_{2}+\frac{1}{a_{3}+\cdots}}}=\left[a_{0} ; a_{1}, a_{2}, a_{3}, \ldots\right],
$$

where $a_{n}$ are integers. If $a_{n}$ is positive for $n \geq 1$ this is called the simple continued fraction expansion of $x$, and this expansion is unique.

A generalized continued fraction expansion is written

$$
x=b_{0}+\frac{a_{1}}{b_{1}+\frac{a_{2}}{b_{2}+\frac{a_{3}}{b_{3}+\cdots}}}=b_{0}+\frac{a_{1}}{b_{1}+\frac{a_{2}}{b_{2}+}} \frac{a_{3}}{b_{3}+} \frac{a_{4}}{b_{4}+} \cdots,
$$

where $a_{n}$ and $b_{n}$ are integers and $a_{n} \neq 0$. By truncating the expansion, we obtain the convergents

$$
r_{n}=b_{0}+\frac{a_{1}}{b_{1}+} \frac{a_{2}}{b_{2}+} \frac{a_{3}}{b_{3}+} \frac{a_{4}}{b_{4}+} \cdots \frac{a_{n}}{b_{n}} .
$$

We write $r_{n}=p_{n} / q_{n}$, with $p_{n}$ and $q_{n}$ coprime integers and define the starting values

$$
p_{-1}=1, \quad q_{-1}=0, \quad p_{0}=b_{0}, \quad q_{0}=1 .
$$

Then, $p_{k}$ and $q_{k}$ for $k \geq 1$ are given by recurrence relations:

$$
p_{k}=b_{k} p_{k-1}+a_{k} p_{k-2}, \quad q_{k}=b_{k} q_{k-1}+a_{k} q_{k-2},
$$

which may be proved by induction (Jones \& Thron, 1980, pg. 20).

This process can be inverted: given a sequence of numerators $p_{n}$ and denominators $q_{n}$ (or just their ratios, the convergents $r_{n}=p_{n} / q_{n}$ ), we can solve (3) for $a_{n}$ and $b_{n}$ :

$$
a_{n}=\frac{p_{n-1} q_{n}-p_{n} q_{n-1}}{p_{n-1} q_{n-2}-p_{n-2} q_{n-1}}, \quad b_{n}=\frac{p_{n} q_{n-2}-p_{n-2} q_{n}}{p_{n-1} q_{n-2}-p_{n-2} q_{n-1}}
$$


together with the starting values $b_{0}=p_{0}, a_{1}=\left(p_{1}-b_{0} q_{1}\right)$ and $b_{1}=q_{1}$.

\section{Continued Fractions for $e$}

From the limit expression $e=\lim _{n \rightarrow \infty}(1+1 / n)^{n}$, Euler's number is the limit of the sequence

$$
\frac{2^{1}}{1^{1}}, \frac{3^{2}}{2^{2}}, \frac{4^{3}}{3^{3}}, \ldots, \frac{(n+1)^{n}}{n^{n}}, \ldots
$$

The terms may be regarded as the convergents of a continued fraction,

$$
r_{n}=\frac{p_{n}}{q_{n}}, \quad \text { where } \quad p_{n}=(n+1)^{n} \quad \text { and } \quad q_{n}=n^{n} .
$$

We can generate a continued fraction by using (4). It begins as

$$
1+\frac{1}{1-} \frac{1}{5-} \frac{13}{10-} \frac{491}{196-} \frac{487903}{9952-} \frac{2384329879}{958144-} \cdots .
$$

The error of this expansion $\left(\log _{10}\left|r_{n}-e\right|\right)$ as a function of truncation is shown in Fig. 1 (dashed line). It is clear that the convergence is very slow.

Euler made extensive studies of continued fractions. For example, his 50-page paper Observations on continued fractions (Euler, 1750) contains numerous original results. One of his best-known expansions is

$$
e=[2 ; 1,2,1,1,4,1,1,6,1,1,8, \ldots] .
$$

The error of Euler's expansion is shown in Fig. 1 (dotted line). It converges much faster than (5). There is a clear signal of period 3, consistent with the recurring pattern $(1,1, n)$ in $(6)$.

\section{CONTINUED FRACTION FROM DERANGEMENT NUMBERS}

A beautiful continued fraction emerges from the relationship between arrangements and derangements. We saw above that

$$
\frac{\text { Arrangements of } n \text { elements }}{\text { Derangements of } n \text { elements }}=\frac{n !}{! n} \rightarrow e .
$$

If we define the numerators and denominators of convergents to be

$$
p_{n}=n ! \text { and } q_{n}=! n,
$$

we can solve for the factors $a_{n}$ and $b_{n}$. The starting values $p_{0}=1, p_{1}=1, q_{0}=1, q_{1}=0$ yield $a_{0}=0, b_{0}=1, a_{1}=1, b_{1}=0$. Then (4) may be solved to yield $a_{n}=b_{n}=n-1$ for $n \geq 2$. Thus we get the expansion

$$
e=1+\frac{1}{0+} \frac{1}{1+} \frac{2}{2+} \frac{3}{3+} \frac{4}{4+} \cdots .
$$

A small adjustment enables us to write this in the elegant form

$$
e=2+\frac{2}{2+} \frac{3}{3+} \frac{4}{4+} \frac{5}{5+} \frac{6}{6+} \cdots .
$$

The error of (7) is shown in Fig. 1 (solid line). Convergence is more rapid than for the other two expansions. 


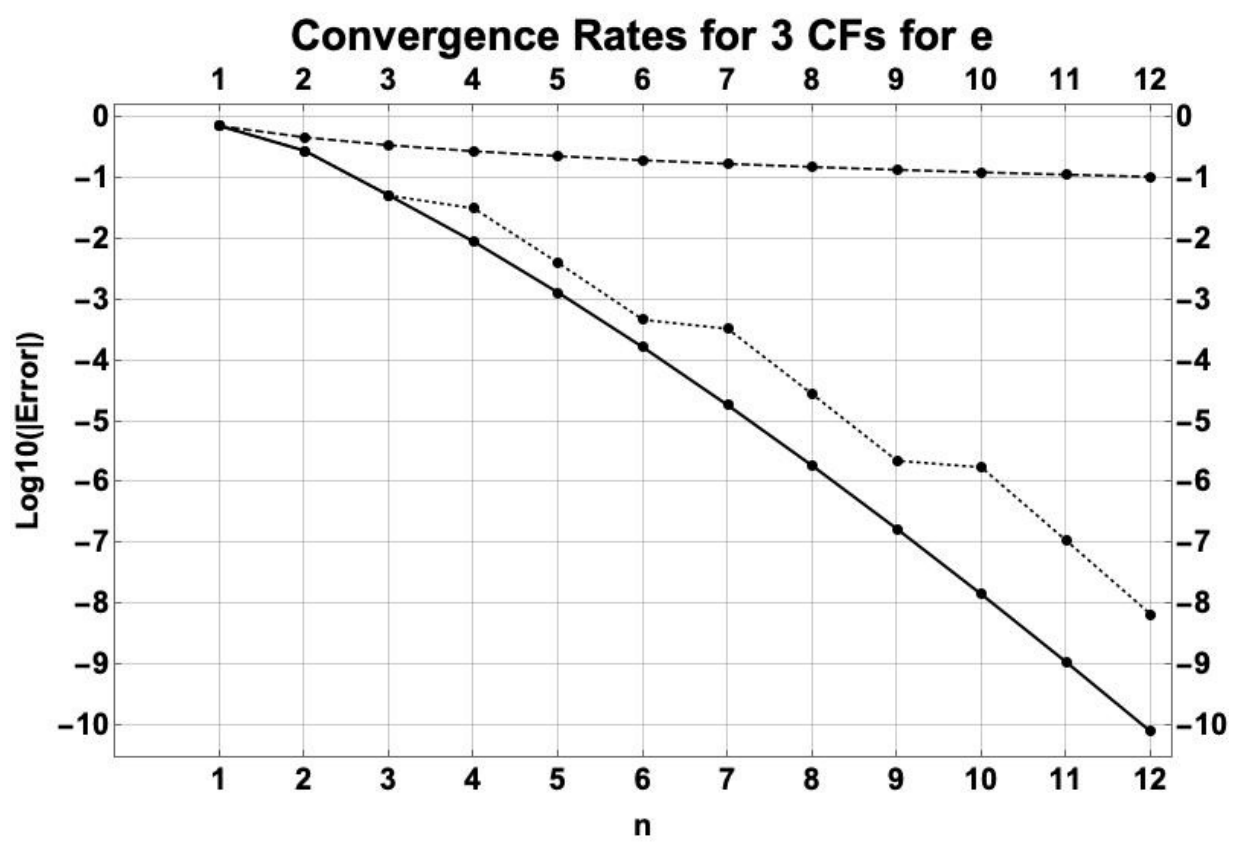

FiguRE 1. Logarithm of the error $\log _{10}\left|r_{n}-e\right|$ in the continued fraction expansions for $e$. Dashed line: $r_{n}=(1+1 / n)^{n}$, Eq. (5). Dotted line: Convergents of Euler's expansion (6). Solid line: $r_{n}=(n+1) ! / !(n+1)$, Eq. (7).

\section{The Ramanujan Machine}

An Automated Conjecture Generator (ACG) called The Ramanujan Machine ${ }^{1}$ has been implemented by a team of mathematicians at the Israel Institute of Technology. This ACG system is capable of producing conjectures about mathematical (and physical) constants, expressed in the form of continued fractions, using only numerical data as input. A paper describing the system is available on the arXiv preprint server (Raayoni, et al., 2020).

The Ramanujan Machine comprises algorithms designed to discover new conjectures, running on a network of computers. The goal of the project is to formulate conjectures that may then be proved mathematically. The ACG has already generated a number of very interesting new conjectures, as well as reproducing several results that were already well known. The website (http://www.ramanujanmachine.com/) enables researchers to submit proofs of conjectures, code new algorithms and (if they wish) allow access to their computers for distributed computation.

While the Ramanujan Machine generates conjectures but not proofs, it has inspired a complementary project using symbolic rather than numerical computation. DoughertyBliss and Zeilberger (2020) describe a system that generates automatic proofs of continued fraction expansions. Their system produced some infinite families of expansions together with rigorous proofs of their validity.

One of the continued fractions discovered by the Ramanujan Machine is

$$
\frac{1}{e-1}=\frac{1}{1+} \frac{2}{2+} \frac{3}{3+} \frac{4}{4+} \frac{5}{5+} \frac{6}{6+} \cdots
$$

\footnotetext{
${ }^{1}$ G. H. Hardy, in his Introduction to Ramanujan's Collected Papers (1927), wrote that Ramanujan's mastery of continued fractions was "beyond that of any mathematician in the world".
} 
which is easily seen to be equivalent to (7) above. This is indicated in Raayoni, et al. (2020) as a "known" result. A proof was presented by Kadyrov and Mashurov (2019). $\mathrm{Lu}$ (2019) gave elementary proofs of other generalized continued fraction formulae for $e$.

The connection with derangement numbers was not made by any of these authors. However, Balof and Jenne (2014) analysed the continued fraction

$$
e=2+\frac{1}{1+} \frac{1}{2+} \frac{2}{3+} \frac{3}{4+} \frac{4}{5+} \cdots,
$$

which was first derived by Euler, and they presented a combinational interpretation of the expansion in terms of derangements.

\section{ACKNOWLEDGEMENT}

I am grateful to an anonymous reviewer, who informed me about the paper of Balof and Jenne (2014) and whose comments helped me to improve the presentation of this work.

\section{REFERENCES}

[1] Balof, Barry and Helen Jenne, 2014: Tilings, continued fractions, derangements, scramblings and e. Journal of Integer Sequences, 17, Art. 14.2.7.

[2] Dougherty-Bliss, Robert and Doron Zeilberger, 2020: Automatic conjecturing and proving of exact values of some infinite families of infinite continued fractions. https://arxiv.org/pdf/2004. 00090.pdf.

[3] Euler, L., 1750: De fractionibus continuis observationes. Commentarii academice scientiarum Petropolitance, 11, 32-81. Reprinted in Opera Omnia, Series 1, 14, 291-349. Translation by Alexander Aycock: Observations on continued fractions. https://arxiv.org/pdf/1808.07006.pdf

[4] Gorroochurn, Prakash, 2012: Classic Problems of Probability. Wiley, ISBN: 978-1-118-06325-5

[5] G. H. Hardy, G. H., P. V. Seshu Aiyar and B. M. Wilson, 2015: Collected Papers of Srinivasa Ramanujan. Cambridge Univ. Press, 392 pp. ISBN: 978-1-1075-3651-7

[6] Jones, William B. and W. J. Thron, 1980: Continued Fractions: Analytic Theory and Applications. Encyclopedia of Mathematics and its Applications. No. 11. Addison-Wesley. ISBN 0-201-13510-8.

[7] Kadyrov, Shirali and Farukh Mashurov, 2019: Generalized continued fraction expansions for $\pi$ and e. https://arxiv.org/pdf/1912.03214.pdf

[8] Lu, Zhentao, 2019: Elementary proofs of generalized continued fraction formulae for $e$. https: //arxiv.org/pdf/1907.05563.pdf

[9] Raayoni, Gal, Shahar Gottlieb, George Pisha, Yoav Harris, Yahel Manor, Uri Mendlovic, Doron Haviv, Yaron Hadad and Ido Kaminer, 2020: The Ramanujan Machine: Automatically generated conjectures on fundamental constants. https://arxiv.org/pdf/1907.00205.pdf

[10] Slone, N. J. A: Sequence A000166 in the Online Encyclopedia of Integer Sequences. https://oeis. org/A000166

[11] Zeilberger, Doron, 2008: Enumerative and algebraic combinatorics. §IV.18 in The Princeton Companion to Mathematics, Ed. Timothy Gowers. Princeton Unversity Press (pp. 550-561). ISBN: 978-0-6911-1880-2.

Peter Lynch is emeritus professor at UCD. His interests include all areas of mathematics and its history. He writes an occasional mathematics column in The Irish Times and has published two books of articles, entitled Thats Maths, Vols I and II. His blog is at http://thatsmaths.com.

School of Mathematics \& Statistics, University College Dublin

E-mail address: Peter.Lynch@ucd.ie 